\section{The 'nivolution' in renal cell carcinoma: behind the scenes of clinical trials}
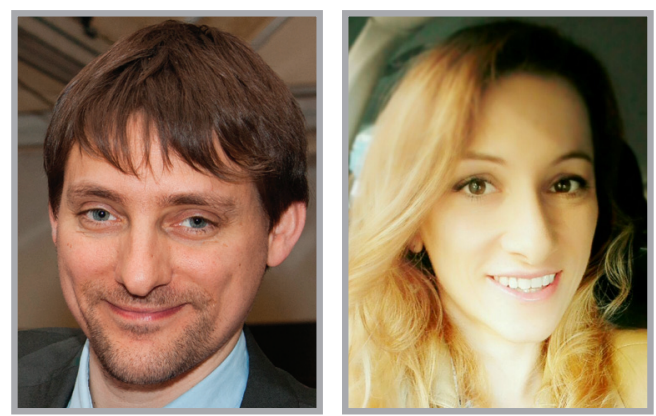

"The benefit of nivolumab treatment was clear in all subgroups compared with everolimus, with a better improvement for poor-risk patients..."

Sebastiano Buti*, \& Melissa Bersanelli

First draft submitted: 21 January 2016; Accepted for publication: 26 April 2016; Published online: 11 May 2016

From the time of IL- 2 and IFN- $\alpha$, much water has flowed beneath the bridge of immunotherapy for renal cell carcinoma (RCC). In the heart of 2015, a 'nivolution' knocked on the doors of this disease: the new immunotherapy with nivolumab, an immune-checkpoint inhibitor against PD-1 receptor, was approved for second line treatment of metastatic RCC (mRCC) according to the results of Phase I, Phase II and Phase III studies [1-3].

\section{Phase I \& II trials}

With an incredible debut, nivolumab obtained an objective response rate (ORR) of $29 \%$ and a median progressionfree survival (PFS) of 7.3 months in the Phase I trial, despite a heavily pretreated population [1].

In the Phase II study, the median overall survival (OS) reached 23 months, longer than those expected among previously treated patients (a third of whom received at least three lines of systemic therapy) [2].

Since we can observe an ORR of 20-22\% and a median PFS of 2.7-4.2 months in the
Phase II trial, it is possible to highlight an unexpected discrepancy between Phase II and Phase I trial. This could provide the opportunity for some speculations, despite the obvious limitations of the comparison among different studies.

First, the ORR may have been affected by treatment regimen since the proposed schedule was different in the two studies: 1 or $10 \mathrm{mg} / \mathrm{kg}$ every 2 weeks in the Phase I, from $0.3,2$ or $10 \mathrm{mg} / \mathrm{kg}$ every 3 weeks in the Phase II trial. Moreover, in the Phase III trial, the subsequently adopted regimen consisted of a further different dose (3 mg/kg every 2 weeks) [3].

Secondly, the Memorial Sloan-Kettering Cancer Center (MSKCC) risk group, used in the Phase II study, was not employed in the Phase I trial, preventing the evaluation of whether the stratification of patients according to the risk group might impact on the results.

Third, a surprisingly large fraction of patients was pretreated with chemotherapy in the Phase I study population (56\%). Considering the great impact of cytotoxic

'Medical Oncology, University Hospital of Parma, Italy

*Author for correspondence: sebabuti@libero.it

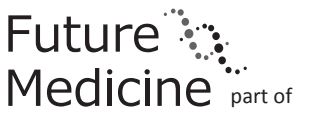

\section{KEYWORDS}

- anti-PD-1 • CheckMate 025

- immune-checkpoint inhibitors

- immunotherapy • kidney cancer

- nivolumab - renal cell carcinoma

- therapy

“...immunotherapy with nivolumab, an immune-checkpoint inhibitor against PD-1 receptor, was approved for second line treatment of metastatic renal cell carcinoma according to the results of Phase I, Phase II and Phase III studies." 
“The great hope in the 'nivolution' for metastatic renal cell carcinoma treatment finally came from the CheckMate 025 Phase III study." drugs on the tumor microenvironment, on the immune system and on DNA repair pathways, a potential sensitizing effect of chemotherapy to immune checkpoint modulators such as nivolumab could be hypothesized. The strict correlation between DNA repair pathway mutations and the efficacy of antibodies targeting PD-1 have been recently demonstrated [4]. In the light of these aspects, considering chemotherapy a still plausible therapeutic choice for selected patients with mRCC [5], it would be interesting to have data about prior cytotoxic treatment also in the Phase II-III study population: these could be useful in order to determine whether pretreatment with chemotherapy could play a positive predictive role respect to nivolumab efficacy.

Finally, a greater rate of stable disease has been obtained in the Phase II study (37-44 vs $27 \%$ of the Phase I): perhaps the disease control rate, which is in fact similar in the Phase I trial, could better explain the OS benefit respect to the ORR. Moreover, the median response duration within the Phase II responders (22 months) was more than doubled compared with those of the more numerous Phase I responding patients. These apparent discrepancies could be partially attributed to the use of Response Evaluation Criteria In Solid Tumors (RECIST) criteria for the disease assessment during treatment instead of the immune-related response criteria (irRC), similar to what was found with nivolumab and ipilimumab in melanoma [6,7]. In fact, longer PFS values were obtained for each study arm, when the analyses were repeated using the irRC assessment (as showed in the appendix of the Phase II study). On the other hand, the lower dose that was chosen for nivolumab in the Phase III study, compared with the previous trials here considered, perhaps could explain the shorter response duration of 12 months.

\section{Phase III trial}

The great hope in the 'nivolution' for mRCC treatment finally came from the CheckMate 025 Phase III study [3]. In this trial, despite a significant advantage for nivolumab in OS (primary end point of the study), reaching 25 months (median value) with early relevant and sustained separation of curves, no improvement was demonstrated for this drug in terms of median PFS, 4.6 months, with late separating curves without reaching statistical significance. In the case of immunotherapy the impact of PFS as possible surrogate of OS could be partially hidden behind the use of RECIST criteria, instead of the irRC, for the disease assessment. As stated above, this view is supported by the longer PFS values obtained in the Phase II study when repeating the analyses using the irRC [2].

Interestingly, the median OS overruled expectations also for the everolimus arm, moving from the awaited 14-15 months [8] to more than 19 months, suggesting a population of patients with relatively good prognosis. In this study, we curiously noticed the use of alternative MSKCC prognostic criteria, developed basing on three parameters in RCC after progression to cytokine treatment [9], instead of the validated and most used original MSKCC criteria, considering five parameters, or of the International Metastatic Renal Cell Carcinoma Database Consortium prognostic score $[10,11]$. This element could influence the clinical significance of the subgroup analysis recently presented at the 12th American Society of Clinical Oncology Genitourinary Cancers Symposium (Abstract 498), necessitating a more accurate interpretation.

As the PD-L1 expression on tumor cells has proved to be a weak predictor of response, with a very low cut-off $(1 \%)$, we wonder whether the expression of PD-L1 on the immune cells infiltrating the tumor could result in a more promising predictive strategy [12]. Moreover, we are looking at the PD-L1 expression as a dynamic biomarker, not only to be used as a static parameter on the tumor specimens at the diagnosis, but also to be evolutionarily followed over time and to be monitored during treatment.

More recently, a subgroup analysis of this Phase III trial has been presented at the 2016 American Society of Clinical Oncology (ASCO) Genitourinary Symposium Annual Meeting [13]. The benefit of nivolumab treatment was clear in all subgroups compared with everolimus, with a better improvement for poor-risk patients, perhaps underlining a more immunogenic disease in this subgroup. Considering the difficulties in the identification of an immunological biomarker driving the treatment choice, a hypermutational status of the tumor could be hypothesized in this population, favoring the response probability to the PD-1/PD-L1 axis blockade [4]. Tumors with high mutational load, or bearing mismatch repair genes defects, or with microsatellite instability, and again cancers not driven by the $V H L$ loss, should be identified in order to find out more immunogenic tumors candidate to receive treatment with immune-checkpoint inhibitors. 
Phase III trials exploring these drugs in the first-line setting of mRCC are currently ongoing.

Several issues remain outstanding, waiting for multidisciplinary solutions: the predictive factors, to be discussed with the pathologist and the biologist; the pattern of response, to be evaluated with the radiologist; the duration of therapy, to be hypothesized with the immunologist with the aim of balancing a good control of disease with the concrete risk of T-cell exhaustion.

\section{References}

1 McDermott DF, Drake CG, Sznol M et al. Survival, durable response, and long-term safety in patients with previously treated advanced renal cell carcinoma receiving nivolumab. J. Clin. Oncol. doi:10.1200/ JCO.58.1041 (2014) (Epub ahead of print).

2 Motzer RJ, Rini BI, McDermott DF et al. Nivolumab for metastatic renal cell carcinoma: results of a randomized Phase II trial. J. Clin. Oncol. 33, 1430-1437 (2015).

3 Motzer RJ, Escudier B, McDermott DF et al. Nivolumab versus everolimus in advanced renal-cell carcinoma. $N$. Engl. J. Med. 373(19), 1803-1813 (2015).

4 Rizvi NA, Hellmann MD, Snyder A et al. Cancer immunology: mutational landscape determines sensitivity to PD-1 blockade in non-small cell lung cancer. Science 348, 124-128 (2015).

5 Buti S, Bersanelli M, Sikokis A et al. Chemotherapy in metastatic renal cell

\section{Financial \& competing interests disclosure}

The authors have no relevant affliations or financial involvement with any organization or entity with a financial interest in or financial conflict with the subject matter or materials discussed in the manuscript. This includes employment, consultancies, honoraria, stock ownership or options, expert testimony, grants or patents received or pending, or royalties.

No writing assistance was utilized in the production of this manuscript. carcinoma today? A systematic review. Anticancer Drugs 24, 535-554 (2013).

6 Eisenhauer EA, Therasse P, Bogaerts J et al. New response evaluation criteria in solid tumours: revised RECIST guideline (version 1.1). Eur. J. Cancer 45(2), 228-247 (2009).

7 Wolchok JD, Hoos A, O'Day S et al. Guidelines for the evaluation of immune therapy activity in solid tumors: immunerelated response criteria. Clin. Cancer Res. 15(23), 7412-7420 (2009).

8 Motzer RJ, Escudier B, Oudard S et al. Efficacy of everolimus in advanced renal cell carcinoma: a double-blind, randomised, placebo-controlled Phase III trial. Lancet 372, 449-456 (2008).

9 Motzer RJ, Bacik J, Schwartz LH et al. Prognostic factors for survival in previously treated patients with metastatic renal cell carcinoma. J. Clin. Oncol. 22, 454-463 (2004).
10 Motzer RJ, Mazumdar M, Bacik J et al. Survival and prognostic stratification of 670 patients with advanced renal cell carcinoma. J. Clin. Oncol. 17, 2530-2540 (1999).

11 Heng DYC, Xie W, Regan MM et al. Prognostic factors for overall survival in patients with metastatic renal cell carcinoma treated with vascular endothelial growth factor-targeted agents: results from a large, multicenter study. J. Clin. Oncol. 27, 5794-5799 (2009).

12 Herbst RS, Soria JC, Kowanetz M et al. Predictive correlates of response to the anti-PD-L1 antibody MPDL3280A in cancer patients. Nature 515, 563-567 (2014).

13 Motzer RJ, Sharma P, McDermott DF et al et al. CheckMate 025 Phase III trial: outcomes by key baseline factors and prior therapy for nivolumab (NIVO) versus everolimus (EVE) in advanced renal cell carcinoma (RCC). J. Clin. Oncol. 34(Suppl. 2S), Abstract 498 (2016). 